\title{
Divertículo de Zenker. Manejo endoscópico contra técnica abierta. ¿Cuál es mejor? Experiencia en el Hospital General de México Dr. Eduardo Liceaga
}

\author{
Zenker's diverticulum: endoscopic versus surgical approach. Which is better? Experience \\ in the General Hospital of Mexico Dr. Eduardo Liceaga
}

Ansony R. Godinez-Vidal*, Luis M. Hurtado-López, Erich O.P. Basurto-Kuba, Edgar Montes de Oca-Durán, Francisco R. Higuera-Hidalgo y Agustín Etchegaray-Donde

Departamento de Cirugía General, Hospital General de México Dr. Eduardo Liceaga, Ciudad de México, México

\begin{abstract}
Resumen
Introducción: El divertículo de Zenker es una enfermedad rara en la población general. Su tratamiento puede llevarse a cabo mediante un enfoque endoscópico o quirúrgico. Objetivo: Reportar la experiencia en el manejo del divertículo de Zenker en un centro de enseñanza de tercer nivel. Métodos: Estudio retrospectivo, transversal, descriptivo, en el que fueron analizados los expedientes de todos los pacientes con diagnóstico de divertículo de Zenker desde la formación de la clínica de tracto digestivo superior del Hospital General de México Dr. Eduardo Liceaga. Resultados: Se encontraron 14 casos con diagnóstico de divertículo de Zenker, 10 tratados con técnica transoral y 4 por cirugía abierta. Se presentaron tres recidivas en la técnica transoral y ninguna con la técnica abierta. En cuanto a las complicaciones, hubo una lesión dental con la técnica transoral y una fístula esofágica con cirugía abierta, que fue manejada con sonda nasoyeyunal colocada por endoscopia hasta el cierre espontáneo de la fístula. Conclusión: El grapado transoral es una técnica con buenos resultados estéticos, pero en nuestra experiencia presenta mayor recurrencia que la cirugía abierta, por lo que debemos realizar un seguimiento a largo plazo de nuestros pacientes.
\end{abstract}

PALABRAS CLAVE: Divertículo de Zenker. Manejo transoral. Manejo endoscópico. Cirugía abierta.

\begin{abstract}
Background: Zenker diverticulum is a rare disease in the general population. Its treatment can be carried out by either an endoscopic or surgical approach. Objective: To report the experience in the management of the Zenker diverticulum in a tertiary education center. Methods: Retrospective, cross-sectional, descriptive study in which the files of all patients with diagnosis of Zenker diverticulum were analyzed from the formation of the upper digestive tract clinic of the General Hospital of Mexico Dr. Eduardo Liceaga. Results: We found 14 cases with diagnosis of Zenker diverticulum, 10 treated with transoral technique and 4 for open surgery. Three recurrences in the transoral technique, no relapse with open technique. In terms of complications, one dental lesion was presented in the technique transoral, and one esophageal fistula in open surgery, managed with a nasojejunal tube placed by endoscopy until the spontaneous closure of said fistula. Conclusions: Transoral stapling is a technique with good aesthetic results. However, in our experience, it has a greater recurrence than open surgery, which is why we must carry out a long-term follow-up of our patients.
\end{abstract}

KEY WORDS: Zenker diverticulum. Transoral management. Endoscopic management. Open surgery.

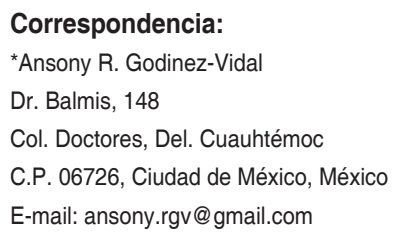

Fecha de recepción: 05-03-2018

Fecha de aceptación: 17-04-2018 DOI: $10.24875 / C I R U . M 18000037$
Cir Cir. 2018;86:244-249

Contents available at PubMed www.cirugiaycirujanos.com 


\section{Introducción}

El divertículo de Zenker o hipofaríngeo es una herniación verdadera de la mucosa esofágica a través de una zona de debilidad que se conoce como «triángulo anatómico de Killian», y que se ubica entre las fibras oblicuas del constrictor inferior de la faringe y las transversales del músculo cricofaríngeo. Se denomina también divertículo falso debido a que sus paredes carecen de muscular, pues solo está formado por mucosa y submucosa'. Aunque fue descrito por primera vez por Abraham Ludlow en $1764^{2}$, debe su nombre al anatomista alemán Friedrich Albert von Zenker, que en 1877 hizo la primera descripción detallada de estos divertículos ${ }^{3}$.

El divertículo de Zenker es una enfermedad rara en la población general. Su tratamiento puede llevarse a cabo mediante un enfoque endoscópico o quirúrgico. La literatura actual favorece el uso de varios procedimientos endoscópicos sobre las técnicas quirúrgicas externas, argumentando que los abordajes endoscópicos reducen el tiempo intraoperatorio y la anestesia, la duración de la estancia hospitalaria y los días hasta que se reinicia la dieta oral. Sin embargo, tales técnicas a menudo tienen mayores tasas de recurrencia sintomática y requieren intervenciones adicionales. Debido a nuestra experiencia con la diverticulectomía endoscópica y abierta, intentamos comparar estos dos procedimientos. En el Hospital General de México se realizó una revisión de 6500 expedientes, donde se hace mención de tres casos, por lo que pensamos que en México es menos frecuente que en la población europea, en la que es más frecuente en hombres y en personas de edad avanzada (séptima a novena décadas de la vida) ${ }^{4}$.

\section{Patología}

Cuando se estudia la pared del divertículo se comprueba que es fina, pero resistente; está constituida por la mucosa, algunas fibras musculares aisladas y una capa fibrosa. En el músculo cricofaríngeo suelen hallarse alteraciones en las fibras musculares, que muestran en partes signos de degeneración y su reemplazo por tejido fibroadiposo5; no se sabe si estas alteraciones son causa o consecuencia de la disfunción muscular. El piso del divertículo, constituido por epitelio pavimentoso, puede mostrar cambios inflamatorios tanto agudos como crónicos, con infiltrados celulares y aumento de la vascularidad.

\section{Fisiopatología}

El aumento de la presión endofaríngea que provoca la protrusión de la mucosa a través del triangulo de Killian puede tener su origen en un asincronismo entre la contracción de las paredes y la relajación del esfínter esofágico superior. Esta hipótesis es la más aceptable habida cuenta de que la incidencia de este divertículo es mayor en personas de más de 50 años, quienes suelen presentar algún grado de deterioro en su coordinación neuromuscular.

Han sido mencionados otros mecanismos fisiopatológicos, tales como ausencia de la relajación del cricofaríngeo (acalasia) 6 , hipertensión aislada del mismo e influencia del reflujo gastroesofágico provocando un espasmo del cricofaríngeo, teniendo en cuenta que la hernia hiatal con reflujo está asociada al divertículo en una proporción que oscila entre el 39 y el 50\%7. No es clara la relación del divertículo de Zenker con la hernia hiatal y el reflujo; recientemente se ha postulado que el reflujo ácido provocado en animales de laboratorio ${ }^{8}$ determina un acortamiento del esófago, y esto genera una fuerte tracción sobre el cricofaríngeo, separándolo del constrictor inferior de la faringe y ampliando la zona de debilidad por donde emergería el divertículo. Esta es una hipótesis interesante que abriría el camino para más investigaciones y extrapolar sus conclusiones a los humanos?.

La realidad es que no existe una frecuencia regular en la concomitancia de ambas patologías, y es un hecho de observación que en los pacientes con grandes divertículos no siempre se comprueba reflujo importante asociado. También se ha observado que algunos enfermos con reflujo mejoran luego de extirpado el divertículo y seccionado el cricofaríngeo, sin observar en estos casos fenómenos aspirativos, como sería de esperar si dicho esfínter ejerciera una función de barrera ${ }^{10}$.

\section{Objetivo}

Este estudio reporta la experiencia en el manejo del divertículo de Zenker en el Hospital General de México Dr. Eduardo Liceaga.

\section{Métodos}

Estudio retrospectivo, descriptivo, transversal, en el que fueron analizados los expedientes de todos los pacientes con diagnóstico de divertículo de Zenker de 
la clínica de tracto digestivo superior del Hospital General de México Dr. Eduardo Liceaga.

\section{Resultados}

Se encontraron 14 casos con diagnóstico de divertículo de Zenker, 10 tratados con técnica transoral y cuatro por cirugía abierta. Se presentaron tres recidivas en la técnica transoral y una con la técnica abierta. En cuanto a las complicaciones, hubo una lesión dental con la técnica transoral y una fístula esofágica con cirugía abierta, la cual se manejó con sonda nasoyeyunal colocada por endoscopia hasta su cierre espontáneo.

\section{Discusión}

Esta es una patología que se puede diagnosticar desde la consulta, pues la presencia de halitosis y el aumento de volumen en el lado izquierdo del cuello nos sugiere la presencia de divertículo de Zenker; algunos pacientes pueden llegar a presentar neumonía de repetición. En nuestra clínica son pocos los pacientes que llegaron con halitosis, la mayoría de ellos presentó disfagia orofaríngea por la compresión que crea el divertículo en la parte proximal del esófago, como consecuencia de la disminución de su luz, y regurgitación de alimentos. Posterior al interrogatorio y la exploración física, con la sospecha diagnóstica, el primer estudio de apoyo diagnóstico es la serie esófago-gastroduodenal con trago de medio hidrosoluble, en la que identificaremos el divertículo en la cara posterior del esófago y observaremos cómo estará comprimiendo su luz y desplazando el esófago (Fig. 1). La endoscopia la utilizamos para excluir alguna otra patología, como puede ser cáncer o alguna afección que cause disfagia. La manometría no la solicitamos de forma sistemática, con excepción de los pacientes con hernia hiatal, con reflujo o cuando sospechamos acalasia. En la endoscopia observamos que la entrada natural del endoscopio es directamente al divertículo, por lo que sugerimos siempre solicitar de manera inicial una serie esófago-gastroduodenal, porque pueden entrar al divertículo sin observar la luz y perforarlo (Fig. 2).

El tratamiento para el divertículo de Zenker debe limitarse a los pacientes sintomáticos. Los objetivos del tratamiento son proporcionar alivio sintomático y mejorar la calidad de vida. El tratamiento consiste en cortar el músculo cricofaríngeo y eliminar el saco reservorio de comida y secreciones, evitando así que

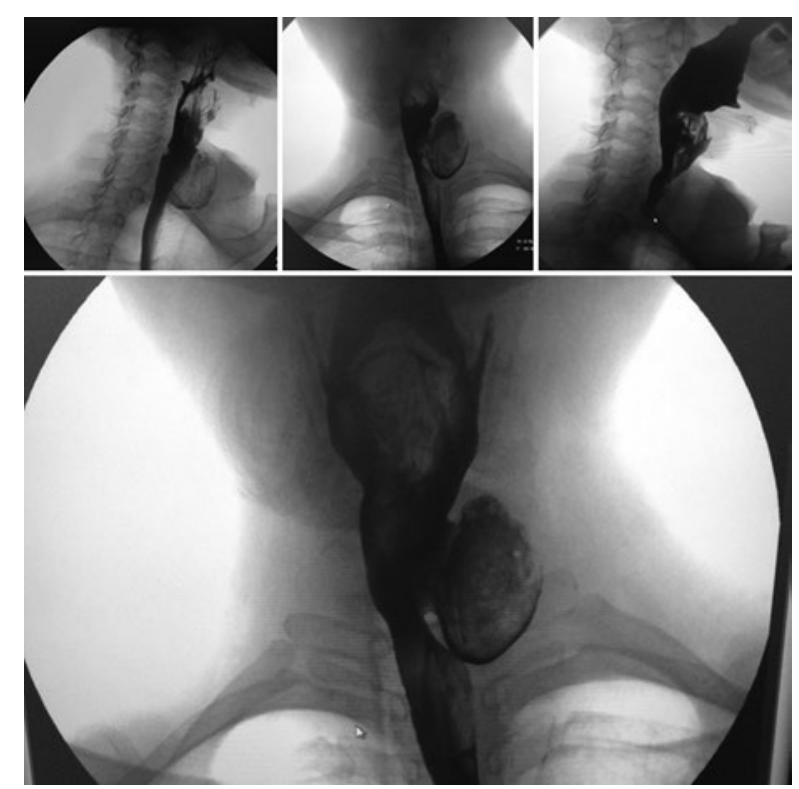

Figura 1. La serie esófago-gastroduodenal es el primer estudio de apoyo diagnóstico, en la que identificaremos el divertículo en la cara posterior del esófago y observaremos cómo estará comprimiendo su luz y desplazando el esófago.

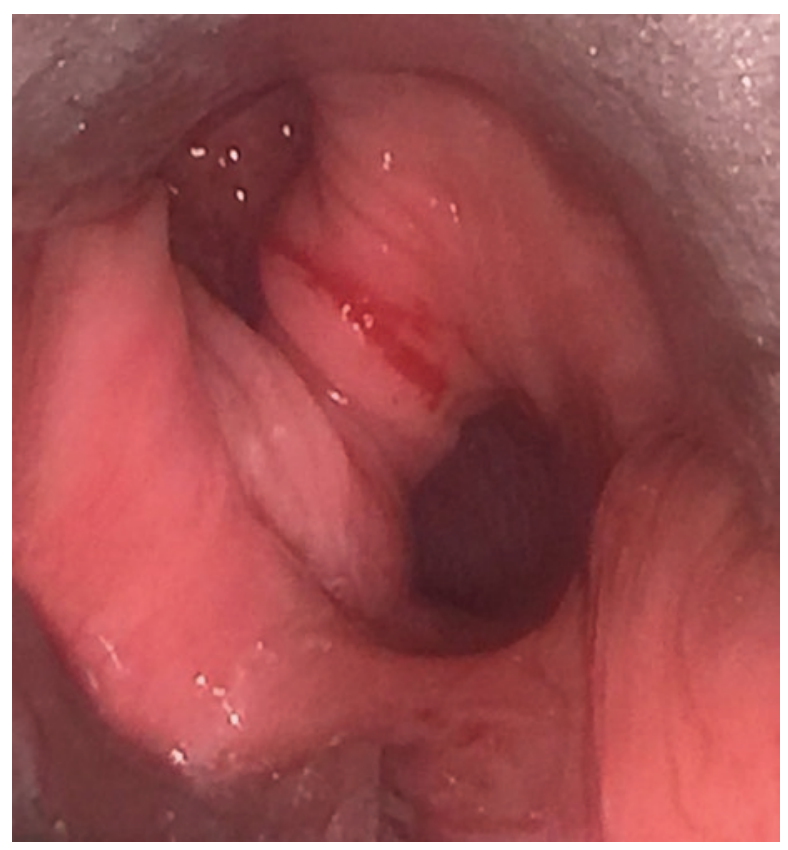

Figura 2. En la endoscopia observamos que la entrada natural del endoscopio es directamente al divertículo. En esta imagen se observa la luz esofágica dividida del divertículo por un septum.

se continúe acumulando. Para ello contamos con diversas técnicas quirúrgicas:

- Diverticulectomía + miotomía abierta.

- Diverticulopexia + miotomía, que se prefiere en divertículos pequeños.

- Miotomía endoscópica.

- Divertículo-esófago anastomosis + miotomía transoral con engrapadora. 


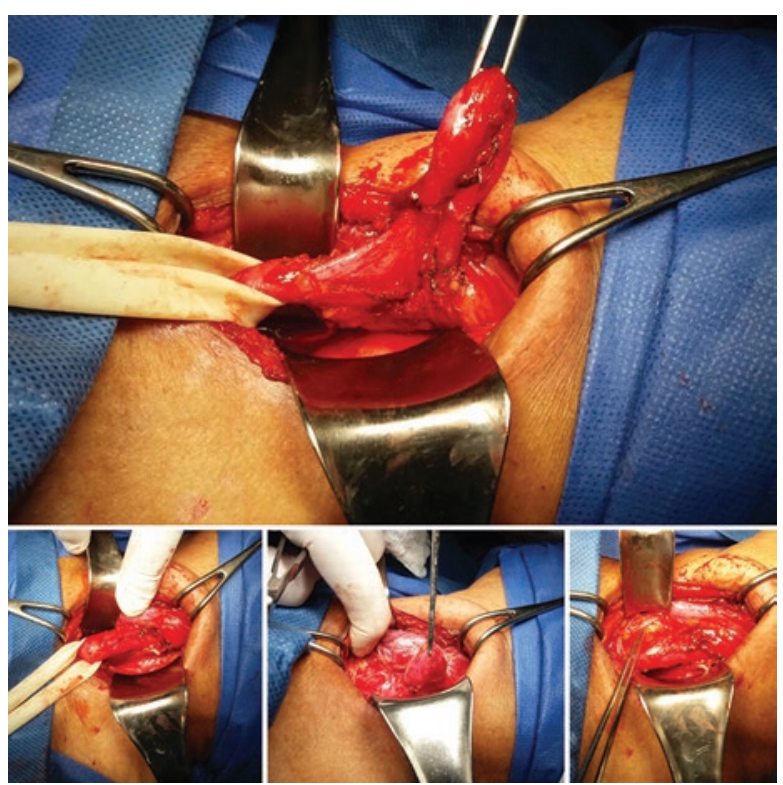

Figura 3. Diverticulectomía con técnica abierta a través de una incisión tipo Koher. El divertículo de Zenker se tracciona con ayuda de la pinza de disección y del Penrose para identificar la base.

\section{Técnica quirúrgica}

En la técnica abierta realizamos una incisión tipo Kocher, lo que nos da una buena exposición y buenos resultados estéticos. Profundizamos por planos hasta llegar a la cara posterior del esófago y la fascia prevertebral, de forma que podamos localizar el divertículo hasta su base y podamos hacer la diverticulectomía de manera adecuada (Fig. 3), y posteriormente la miotomía de unos $3-5 \mathrm{~cm}$. En ocasiones realizamos la diverticulectomía con engrapadora, pero también hemos utilizado sutura. Es importante no traccionar demasiado el divertículo, porque podemos dejar al paciente con estenosis esofágica. Otra opción quirúrgica es la diverticulopexia, en la que se sutura el divertículo en la parte superior, evitando que funcione como un reservorio.

En la técnica endoscópica transoral se requiere colocar al paciente en hiperextensión del cuello, por lo que pacientes de edad avanzada con limitación importante para hiperextender o con disminución de la apertura bucal quedan excluidos de este tipo de manejo. Con el paciente bajo los efectos de la anestesia general, se procede a introducir el laringoscopio. La luz que se logra visualizar es pequeña, por lo que es un procedimiento con alto grado de dificultad (Fig. 4). Cuando observamos el septum entre el divertículo y el esófago, se introduce la engrapadora en dicho septum y se procede a realizar la miotomía completa, con división de la pared común entre el

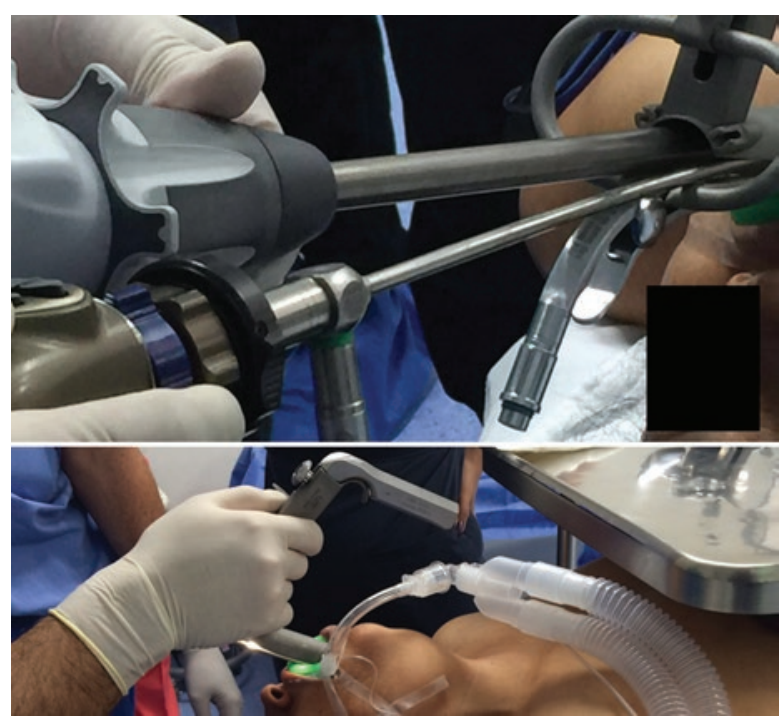

Figura 4. En la técnica endoscópica se requiere colocar al paciente en hiperextensión del cuello. Bajo los efectos de la anestesia general, se procede a introducir el laringoscopio. La luz que se logra observar es pequeña. Cuando vemos el septum entre el divertículo y el esófago, se introduce la engrapadora en el septum.

divertículo y el esófago, seguido por cierre simultáneo inmediato de los bordes divididos con las grapas. Recomendamos colocar dos puntos con el Endo Stitch $^{\mathrm{TM}}$ en el tabique del cricofaríngeo, para poder calzar adecuadamente la engrapadora y estar seguros de que tenemos la totalidad del cricofaríngeo seccionado. Es importante tener en cuenta la longitud del cricofaríngeo, que es de aproximadamente $3 \mathrm{~cm}$; un divertículo menor de $3 \mathrm{~cm}$ contraindica este manejo, por lo que la longitud mayor de la engrapadora haría una miotomía incompleta. La divertículo-esófago anastomosis + miotomía transoral con engrapadora es una técnica con buenos resultados estéticos, ya que no realizamos incisiones cutáneas, no se dejan drenajes, no existe lesión del nervio laríngeo recurrente, presenta menor estancia hospitalaria, y tiene menor morbilidad y mortalidad; sin embargo, está contraindicada en divertículos menores de $3 \mathrm{~cm}$. En cuanto a la duración del procedimiento, en la literatura mundial se reporta un tiempo transquirúrgico de 25 minutos, pero en nuestra experiencia tenemos una duración de la cirugía de hasta 60 minutos, por el tiempo transcurrido en la colocación adecuada del diverticuloscopio y el endoscopio, en comparación con la técnica abierta que reporta una duración de 60 a 90 minutos.

En una serie de casos de 900 pacientes en la Clínica Mayo de 1944 a 1978 se reporta que el tratamiento abierto obtuvo éxito en el $93 \%$, presentando recurrencia el $3.6 \%$, infección el $1.2 \%$, fístula el $1.8 \%$, 
parálisis de cuerda vocal por lesión del nervio laríngeo recurrente el $3.6 \%$, y una mortalidad del $1.2 \%{ }^{11}$.

En Italia, en los años 1990, en una serie de 90 pacientes se muestra la técnica transoral como novedosa, reportando un seguimiento de 23 meses, con poca recidiva, cero morbilidad y cero mortalidad ${ }^{12}$. Muchos autores citan esta técnica como de elección. Recientemente, en el Reino Unido un estudio reporta falla intraoperatoria del $7.7 \%$, complicaciones del $9.9 \%$ y recurrencias del $12 \%{ }^{13}$.

En una revisión sistemática que compara la técnica endoscópica contra la abierta se observa una falla mayor a corto plazo con la técnica transoral, mayor dificultad en obtener un adecuado acceso y menos complicaciones; sin embargo, pueden llegar a tener mediastinitis en un bajo porcentaje, en comparación con la técnica abierta, con la que en ningún caso se tienen problemas en obtener un adecuado acceso al divertículo, pero que presenta mayores complicaciones (como la fistula esofágica), se reporta una disminución de la calidad de vida y los tiempos de estancia hospitalaria son más prolongados ${ }^{14}$. En un metaanálisis de 596 pacientes que comparó abordajes endoscópicos y quirúrgicos, Albers, et al. ${ }^{15}$ informaron una mejoría en la reducción de los síntomas con cirugía (diferencia de medias estandarizada: 0.08; intervalo de confianza [IC] del 95\%: 0.03-1.13), pero mayores tasas de complicaciones (diferencia de medias estandarizada: 0.09; IC 95\%: 0.03-0.43). En particular, las diferencias en el tamaño del divertículo entre los grupos no se sometieron a la evaluación de la heterogeneidad.

La técnica endoscópica transoral es una opción segura, efectiva y mínimamente invasiva para tratar el divertículo de zenker ${ }^{16-19}$, pero solo un estudio ha proporcionado datos a largo plazo ${ }^{20}$.

La popularidad de esta técnica ha catalizado la investigación y el desarrollo de accesorios, tecnologías y enfoques operativos disponibles para maximizar el perfil de seguridad y eficacia. El metaanálisis de Ishaq, et al. ${ }^{21}$ presentó tasas de complicaciones del $11.3 \%$ de los casos, y recurrencia del $11 \%$. Los estudios realizados antes de 2005 se asociaron con un mayor éxito clínico, pero con tasas de recurrencia altas. Esta observación es interesante, en especial cuando la calidad de la capacitación y la práctica han mejorado considerablemente durante este tiempo. Una posible explicación podría ser la falta de una única técnica estandarizada. A medida que la tecnología para la terapia de esta patología evoluciona sin el volumen de pacientes adecuado para desarrollar la experiencia del cirujano, la combinación óptima de técnica y dispositivo para lograr seguridad, eficacia y resultado a largo plazo favorables sigue sin estar clara ${ }^{21}$.

Debido a la infrecuencia relativa de la afección, los estudios para su terapia están, en el mejor de los casos, limitados a pequeños estudios prospectivos, sin datos de ensayos controlados aleatorizados. Existe una clara necesidad de estudios prospectivos comparativos más grandes, bien diseñados, para evaluar el método y el dispositivo de tratamiento óptimos, a fin de permitir la estandarización de la capacitación y la práctica. Además, las definiciones universales de los resultados, como el éxito del tratamiento y las tasas de recaída y de complicaciones, son necesarias para permitir una mejor comparación entre los estudios y para garantizar la calidad de la práctica.

Desde el comienzo de la técnica transoral no ha habido consenso sobre la capacitación y los aspectos técnicos de la terapia. Como se discutió en este estudio, la técnica óptima sigue siendo controvertida; por ejemplo, la importancia de mantener la fascia bucofaríngea intacta durante la incisión del tabique, como recomiendan Chang, et al. ${ }^{22}$, rara vez se ha informado. Debido a que el divertículo de Zenker es un trastorno poco frecuente, el entrenamiento en endoscopia terapéutica puede ser un desafío, con una curva de aprendizaje desconocida. Existe una clara necesidad de garantizar que la capacitación para un procedimiento de alto riesgo haya sido de calidad suficiente, y que se hayan alcanzado las competencias técnicas necesarias antes de permitir la práctica no supervisada. El énfasis debe comenzar con consideraciones anatómicas. Como la mayoría de los cerdos domésticos poseen divertículos faríngeos que se asemejan a los humanos ${ }^{23}$, los modelos porcinos pueden ser una herramienta útil en el entrenamiento y la evaluación. También está en desarrollo un simulador quirúrgico que demuestra el contenido y la validez de constructo al permitir que los residentes adquieran las habilidades dentro de un entorno controlado para desarrollar competencia en la terapia del divertículo de Zenker ${ }^{24}$.

Los criterios que utilizamos en la clínica de tracto digestivo superior para decidir qué tipo de abordaje vamos a ofrecer a nuestro paciente con divertículo de Zenker son la edad (en el paciente joven se prefiere la cirugía abierta), la movilidad del cuello (el paciente con limitación a la hiperextensión se excluye del manejo transoral), la dentadura (por la posibilidad de lesión dental al introducir el laringoscopio) y la existencia del instrumental para poder realizar la técnica transoral. 


\section{Conclusión}

En nuestra institución observamos que el grapado transoral es una técnica con buenos resultados estéticos, con inicio de la vía oral el mismo día de la cirugía y con menor estancia hospitalaria. La muestra es pequeña, pero en la experiencia de la clínica el manejo transoral es una técnica con mayor recurrencia que la cirugía abierta, por lo que sugerimos individualizar cada caso y realizar un seguimiento a largo plazo de los pacientes.

\section{Conflicto de intereses}

No existe ningún conflicto de intereses.

\section{Bibliografía}

1. Herrero A, Pérez L, Tejero-Garcés G, Guallar M, Orte C, Ortiz A. Tratamiento del divertículo de Zenker: comparación de diferentes técnicas. Acta Otorrinolaringol Esp. 2013;64:1-5.

2. Ludlow A. A case of obstructed deglutition, from a preternatural dilatation of, and bag formed in, the pharynx. Med Obs Inq. 1764;3:85-101.

3. Zenker FA, von Ziemssen H. Krankheiten des oesophagus. Handbuch der Speciellen Pathologie und Therapie. 7. Leipzig: Vogel; 1877.

4. Pérez Torres E, Pérez Pineda J. Diagnóstico y tratamiento del divertículo de Zenker. Rev Med Hosp Gen Mex. 2005;68:155-9.

5. Constantini M, Zaninoto G, Rizzetto C, Narne S, Ancona E. Oesophageal diverticula. Best Pract Res Clin Gastroenterol. 2004;18:3-17.

6. Cook IJ, Gabb M, Panagopoulos V, Jamieson GG, Dodds WJ, Dent J, et al. Pharingeal (Zenker's) diverticulum ia a disorder of upper esophageal sphincter opening. Gastroenterology. 1992;103:1229-35.

7. Gage-White L. Incidenca of Zenker's diverticulum with hiatus hernia Laryngoscope. 1998:98:527-30.
8. Paterson WG, Kolyn DM. Esophageal shortening induced by short-term intraluminal acid perfusion in opossum; a cause for hiatus hernia? Gastroenterology. 1994;107:1736-40.

9. Sasaki CT, Ross DA, Hundal J. Association between Zenker diverticulum and gastroesophageal reflux disease: development of working hypotesis. Am J Med. 2003;115:169S-171S.

10. Feussner H, Siewert JR. Zenker's diverticulum and reflux. Hepatogastroenterology. 1992;32:95-6.

11. Payne WS. The treatment of pharyngoesophageal diverticulum: the simple and complex. Hepatogastroenterology. 1992;39:109-14.

12. Peracchia A, Bonavina L, Narne S, Segalin A, Antoniazzi L, Marotta G. Minimally invasive surgery for Zenker diverticulum: analysis of results in 95 consecutive patients. Arch Surg. 1998;133:695-700.

13. Leong SC, Wilkie MD, Webb CJ. Endoscopic stapling of Zenker's diverticulum: establishing national baselines for auditing clinical outcomes in the United Kingdom. Eur Arch Otorhinolaryngol. 2012;269:1877-84.

14. Johnson CM, Postma GN. Zenker diverticulum - which surgical approach is superior? JAMA Otolaryngol Head Neck Surg. 2016;142:401-3.

15. Albers DV, Kondo A, Bernardo WM, Sakai P, Moura RN, Silva GL, et al. Endoscopic versus surgical approach in the treatment of Zenker's diverticulum: systematic review and meta-analysis. Endosc Int Open. 2016;4:E678-86.

16. Law R, Katzka DA, Baron TH. Zenker's diverticulum. Clin Gastroenterol Hepatol. 2014;12:1773-82.

17. Verdonck J, Morton RP. Systematic review on treatment of Zenker's diverticulum. Eur Arch Otorhinolaryngol. 2015;272:3095-107.

18. Antonello A, Ishaq S, Zanatta L, Cesarotto M, Costantini M, Battaglia G. The role of flexible endotherapy for the treatment of recurrent Zenker's diverticula after surgery and endoscopic stapling. Surg Endosc. 2016;30:2351-7.

19. Aiolfi A, Scolari F, Saino G, Bonavina L. Current status of minimally invasive endoscopic management for Zenker diverticulum. World J Gastrointest Endosc. 2015;7:87-93.

20. Bonavina L, Aiolfi A, Scolari F, Bona D, Lovece A, Asti E. Long-term outcome and quality of life after transoral stapling for Zenker diverticulum. World J Gastroenterol. 2015;21:1167-72.

21. Ishaq S, Hassan C, Antonello A, Tanner K, Bellisario C, Battaglia G, et al. Flexible endoscopic treatment for Zenker's diverticulum: a systematic review and meta-analysis. Gastrointest Endosc. 2016;83:1076-89.e5.

22. Chang CWD, Liou SS, Netterville JL. Anatomic study of laser-assisted endoscopic cricopharyngeus myotomy. Ann Otol Rhinol Laryngol. 2005; 114:897-901.

23. Baron TH. Endoscopic management of Zenker diverticula. Gastroenterol Hepatol. 2017;13:242-4.

24. Wiebracht ND, Giliberto JP, Myer C 4th, Casper K, Johnson KE. Pilot testing of a novel surgical simulator for endoscopic Zenker's diverticulotomy. Laryngoscope. 2017;127:592-6. 